\title{
Sensitivity of the Hilbert-Huang Transform to interpolation methodology: examples using synthetic and ocean data
}

\author{
F.M. Ziaeyan Bahri ${ }^{a}$ and J.J. Sharples ${ }^{a}$ \\ ${ }^{a}$ School of Physical, Environmental and Mathematical Sciences, UNSW Canberra, Australia \\ Email: Fatemeh.Ziaeyanbahri@student.adfa.edu.au
}

\begin{abstract}
The Hilbert-Huang Transform (HHT) provides a way to decompose a signal into component functions and to analyse instantaneous frequency data. Moreover, it applies to nonlinear and non-stationary data, and so offers a practical alternative to more traditional methods such as the Fourier transform, which is not well-suited to such circumstances. Since its inception in the late 1990s, the HHT has been applied in a number of areas, including biomedicine, neuroscience, epidemiology, chemical engineering, finance, atmospheric turbulence, seismology and ocean dynamics. The HHT combines a data sifting process known as empirical mode decomposition (EMD) with Hilbert spectral analysis. The EMD process decomposes a signal into intrinsic mode functions (IMFs), which are analogous to the harmonic modes of Fourier analysis, but which can have variable amplitude and frequency throughout the time domain. A critical step in determining each of the IMFs involves constructing upper and lower envelopes of the local maxima and minima. In the original presentation of the HHT methodology, cubic spline interpolation was recommended to construct these envelopes. However, there does not appear to be any literature to support such a recommendation and so it is natural to wonder how employing alternative interpolation methods would affect the ultimate outcome of the HHT method.
\end{abstract}

In this paper we consider this issue by studying the HHT, and in particular the EMD procedure, as it applies to two synthetic data sets (one a noisy realisation of the other) and to a real geophysical data set, namely sea level data collected by a tidal gauge at Brisbane. IMFs for each of the data sets are derived separately using cubic spline and linear interpolation techniques and the resulting structures scrutinized for systematic differences.

The analyses of the synthetic data sets showed only minor differences between the results produced by the linear and cubic interpolation methods, with the linear interpolation method producing the most accurate of the underlying trend in the input signal. The cubic interpolation EMD method produced better estimates of oscillatory signal components, though these estimates suffered from larger errors near the boundaries when compared with those obtained from the linear EMD procedure.

Analyses of the noisy synthetic data set again showed only relatively minor differences in the performance of linear and cubic ensemble EMD. Both methods were affected by mode mixing issues, but the linear method again provided the more accurate estimate of the trend in the signal. The low frequency oscillatory component of the signal was estimated more accurately by the cubic ensemble EMD method, while the high frequency component was reproduced to a similar degree of accuracy by both linear and cubic methods. Overall, the choice of interpolation method used when applying the EMD procedure to the synthetic data sets only produced minor changes in the output.

Using the linear and cubic methods to estimate the long term trend in sea level at Brisbane produced similar results, but with differences that could be considered significant in the context of understanding the impacts of trends in sea level. One key point of difference was that the linear EMD method delivered a trend estimate with discontinuous derivative, which may be considered less desirable in some circumstances.

Keywords: Non-stationary time series, Hilbert-Huang Transform, Empirical Mode Decomposition, interpolation, data analysis, Empirical Mode Decomposition 
F.M. Ziaeyan Bahri and J.J. Sharples, Sensitivity of the Hilbert-Huang Transform to interpolation methodology...

\section{INTRODUCTION}

The need to decompose a time series, or some other signal, into its primitive or fundamental components arises in a number of applications. For example, in electronics signal processing or in certain geophysical applications, insights can be gained by decomposing a complex signal into components with distinct frequency characteristics, which can then be analysed to identify particular driving mechanisms. The Fourier transform is the pervasive example in this respect, though other examples such as principal components analysis and wavelet analysis are also widely employed. While methods like the Fourier transform are key theoretical tools in signal decomposition, they can suffer from certain shortcomings such as being poorly suited to analysis of nonlinear and non-stationary data.

Unlike global methods like the Fourier transform, the empirical mode decomposition (EMD) procedure (Huang et al., 1998) expresses the input time series in terms of an a posteriori defined basis, which is derived from the data itself. The EMD procedure decomposes the input signal into a number of intrinsic mode functions (IMFs). When combined with the Hilbert transform (Hahn, 1996), which delivers information about the frequency/power spectra of the intrinsic modes, EMD constitutes a useful data analysis technique that can be more suitably applied to nonlinear and non-stationary time series. The combination of EMD and Hilbert spectral analysis is referred to collectively as the Hilbert-Huang Transform (HHT).

A number of authors have used the HHT method for the analysis of signals that exhibit complex trend behaviour. Indeed, Ezer et al. (2013) employed the HHT to analyse sea level variability in the western Atlantic ocean and how it related to changes in the Gulf Stream. Similarly, Salisbury and Wimbush (2002) used the HHT method to examine the predictability of ENSO events, while Huang et al. (2001) use the method to analyse seismological data.

Unfortunately the HHT method has a number of drawbacks. These include boundary effects (Dätig and Schlurmann, 2004) and mode mixing (Deering and Kaiser, 2005). Mode mixing occurs when a single IMF consists of signals of widely disparate time scales, or when similar time scale signals reside in different IMF components. The presence of noise in the input signal is known to cause problems with mode mixing in the HHT method (Deering and Kaiser, 2005). To overcome this problem Huang and Wu (2008) introduced the ensemble EMD (EEMD) procedure, which is a noise-assisted version of EMD.

The EMD procedure has an intrinsic dependence on interpolation methodology, which is repeatedly used to obtain upper and lower envelopes of data as one of the sub-procedures of EMD. In the majority of implementations of the HHT/EMD method cubic interpolation is employed. Exceptions include the works of Peel et al. (2009, 2011); Pegram et al. (2008); Srikanthan et al. (2011), in which rational spline interpolation is used as a generalisation of the cubic spline methodology. Peel et al. (2009); Pegram et al. (2008); Srikanthan et al. (2011) investigated the effect of different interpolation methods in EMD applied to rainfall data, while Peel et al. (2011)considered the same for a synthetic data set. In this paper, we complement the aforementioned studies by implementing the EMD procedure with a linear interpolation methodology and comparing its results with the usual cubic interpolation based EMD procedure. The comparison is facilitated using synthetic data, both in the absence and presence of noise, as well as a real world geophysical data set. We note that Rilling et al. (2003) suggested that cubic interpolation performed better than linear interpolation, but this claim was not supported quantitatively. We also note that in this initial investigation we only consider the effect on the IMFs and ensemble IMFs; the effect on the power spectra obtained through Hilbert spectral analysis is not considered.

\section{DATA AND Methods}

\subsection{Empirical mode decomposition and Hilbert-Huang transform}

Huang et al. (1998) show how a time series $y(t)$ can be decomposed into a number of intrinsic mode functions (IMFs), which satisfy certain defining conditions. The IMFs of $y(t)$ are determined through a sifting process known as empirical mode decomposition (EMD). The sifting procedure first identifies all of the local extrema in the signal $y(t)$. The local maxima and minima are then interpolated separately to define upper and lower envelopes of $y$. The mean of the upper and lower envelopes $m_{1}$ is then subtracted from the original time series $y$ to give the first protomode $h_{1}=y-m_{1}$. This procedure is then repeated iteratively, so at the $j$ th iteration we have $h_{j}=h_{j-1}-m_{j}$, where $m_{j}$ is the mean of the upper and lower envelopes of $h_{j-1}$, determined by interpolation of the local maxima and minima. The iteration is continued $k$ times until a stoppage condition is satisfied (Huang and Wu, 2008). The time series $h_{k}$ is identified as the first IMF, denoted $c_{1}$. 
F.M. Ziaeyan Bahri and J.J. Sharples, Sensitivity of the Hilbert-Huang Transform to interpolation methodology...

This process is then applied to the residual time series $r_{1}=y-c_{1}$ to deliver the second IMF $c_{2}$ and the second residual $r_{2}=r_{1}-c_{2}$. Subsequent IMFs $c_{3}, \ldots, c_{n}$ and residuals $r_{3}, \ldots, r_{n}$ are obtained similarly. The sifting process terminates when $r_{n}$ has at most one extremum. The original time series has then been decomposed as follows:

$$
y(t)=\sum_{j=1}^{n} c_{j}(t)+r_{n}(t)
$$

When dealing with noisy data sets the EMD procedure is implemented as part of an ensemble process, which is referred to as ensemble EMD (EEMD). The EEMD procedure is defined algorithmically by the following steps (Huang and Wu, 2008): (1) add white noise to the input time series; (2) use EMD to decompose the input time series with the noise added into IMFs; (3) repeat steps (1) and (2) many times with different random selection of the white noise in each case; (4) obtain the (ensemble) means of corresponding IMFs of the various EMD applications as the final ensemble IMFs.

We note that we have implemented the EMD procedure as given by Huang and Wu (2008). In particular, we have not attempted to ameliorate any of the known issues with boundary effects and extrapolation of the interpolating functions beyond the first and last extreme points. The reader is referred to Dätig and Schlurmann (2004) and Peel et al. (2009) for further discussion of this issue.

\subsection{Synthetic data}

To examine the sensitivity of the EMD procedure to alteration of the interpolation methodology used in its implementation, we consider synthetic time series data $y=\left(y_{1}, y_{2}, \ldots, y_{1000}\right)$ defined by:

$$
y_{t}=1-\frac{t}{2000}+0.4 \sin \left(\frac{2 \pi t}{200}\right)+0.2 \sin \left(\frac{2 \pi t}{25}\right) ; \quad t=1,2, \ldots, 1000 .
$$

This data set is chosen at it represents a non-stationary time series comprised of three well-defined components: a long-term linear trend; and two sinusoidal signals with different frequencies. The relative efficacy of the two EMD procedures based on cubic and linear interpolation we be evaluated based on how well they can reproduce the three component signals.

As noted above, the presence of noise in the input time series can produce mode mixing across the IMFs and the need to employ the ensemble EMD method to achieve reasonable IMFs. To assess the effects of changing the interpolation method in the EEMD procedure when applied to noisy data we also consider the time series $Y=\left(Y_{1}, Y_{2}, \ldots, Y_{1000}\right)$ defined by:

$$
Y_{t}=y_{t}+\epsilon_{t} ; \quad \epsilon_{t} \sim N(0,0.01), \quad t=1,2, \ldots, 1000 .
$$

\subsection{Sea level data}

To test the effects of the different EMD methodologies applied to a data set of particular geophysical interest we consider tidal gauge data recorded at Brisbane, which provides a measure of local sea level. The tidal gauge record at Brisbane is one of the longer records, which makes it useful in applications such as studies of the impacts of climate change on sea level (e.g. Church et al., 2006; Rahmstorf, 2012; Ezer et al., 2013;). The Brisbane sea level data were obtained from the Permanent Service for Mean Sea Level (PSMSL; http://www.psmsl.org/) and spans the period of 1980 to 2011.

\section{RESULTS}

The EMD and EEMD methodologies were applied to the signals (1) and (2), respectively. In each case the methodology was implemented separately using cubic and linear interpolation to construct the upper and lower envelopes. The two approaches were then applied to sea level data and their output compared.

\subsection{Synthetic data}

Figure 2 summarises the results of applying the EMD method to the synthetic signal (1). Figure 2a shows the IMF corresponding to the long term trend in the signal plotted against the (true) linear trend component (i.e. the first two terms on the right of equation 1)). As can be seen the EMD procedure was able to extract the long 
F.M. Ziaeyan Bahri and J.J. Sharples, Sensitivity of the Hilbert-Huang Transform to interpolation methodology...
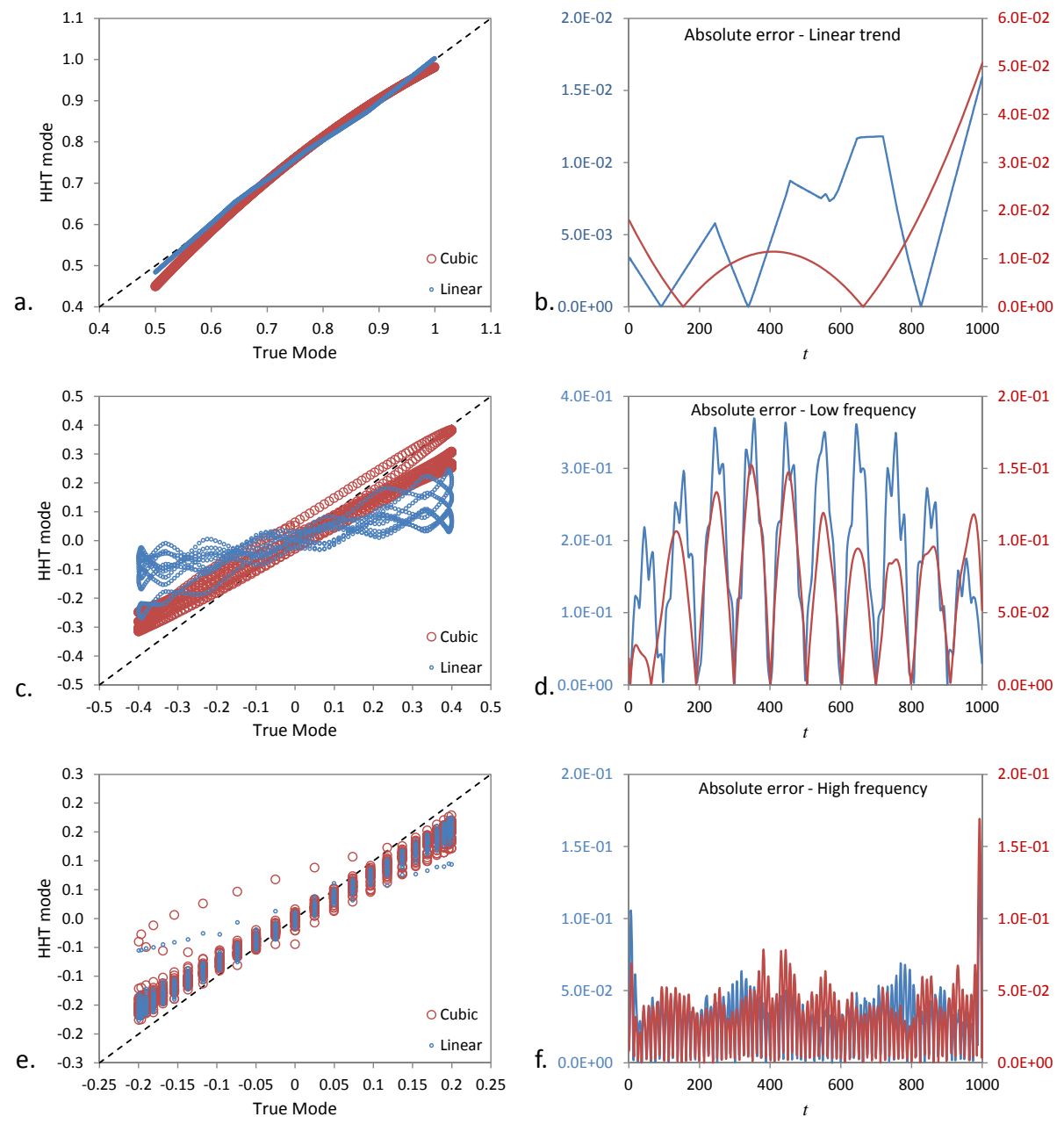

Figure 1. Results for the noisy synthetic data (2). In all figure panels, results pertaining to the linear EMD method are in blue and those pertaining to the cubic EMD method are in red: (a) EMD/HHT trend plotted against the actual trend; (b) Absolute error between modelled and actual trend; (c) EMT/HHT low frequency signal plotted against the actual low frequency component; (d) Absolute errors for the low frequency component; (e) EMT/HHT high frequency signal plotted against the actual high frequency component; (d) Absolute errors for the high frequency component.

term trend with a high degree of accuracy regardless of whether it was based on a linear or cubic interpolation method. Figure $2 \mathrm{~b}$ provides a more detailed appraisal of the accuracy of the two different methods, and indicates that the EMD procedure using a linear interpolation method produces a uniformly smaller absolute error than the cubic interpolation based EMD procedure. For both approaches the largest errors were found at the ends of the domain. The average absolute error for the trend component was $1.2 \times 10^{-4}$ using linear interpolation, and $2.1 \times 10^{-4}$ using cubic interpolation.

Figures $2 \mathrm{c}$ and $2 \mathrm{~d}$ show the corresponding plots for the low frequency component of the input signal (i.e. the thirds term on the right of equation 1)). Again, both the linear and cubic EMD methods are able to accurately reproduce the input signal component, though not as accurately as they were able to resolve the linear trend component. Larger absolute errors were again found at the ends of the domain, though absolute errors arising from the linear EMD method did not exhibit as large an increase in the error at the domain ends as the cubic EMD method. Overall the cubic EMD method produced smaller absolute errors, with a mean absolute error of $5.0 \times 10^{-4}$ compared with $3.2 \times 10^{-3}$ for the linear EMD method. Results for the remaining high frequency component of the input signal (1) were very similar (figures $2 \mathrm{e}$ and $2 \mathrm{f}$ ), with the cubic EMD method producing a mean absolute error of $4.9 \times 10^{-4}$ compared with $3.2 \times 10^{-3}$ for the linear EMD method.

The results of applying the linear and cubic EEMD procedures to the noisy synthetic signal (2) are summarised in Figure 1. The EEMD method was implemented by adding a noise component with a standard deviation 
F.M. Ziaeyan Bahri and J.J. Sharples, Sensitivity of the Hilbert-Huang Transform to interpolation methodology...
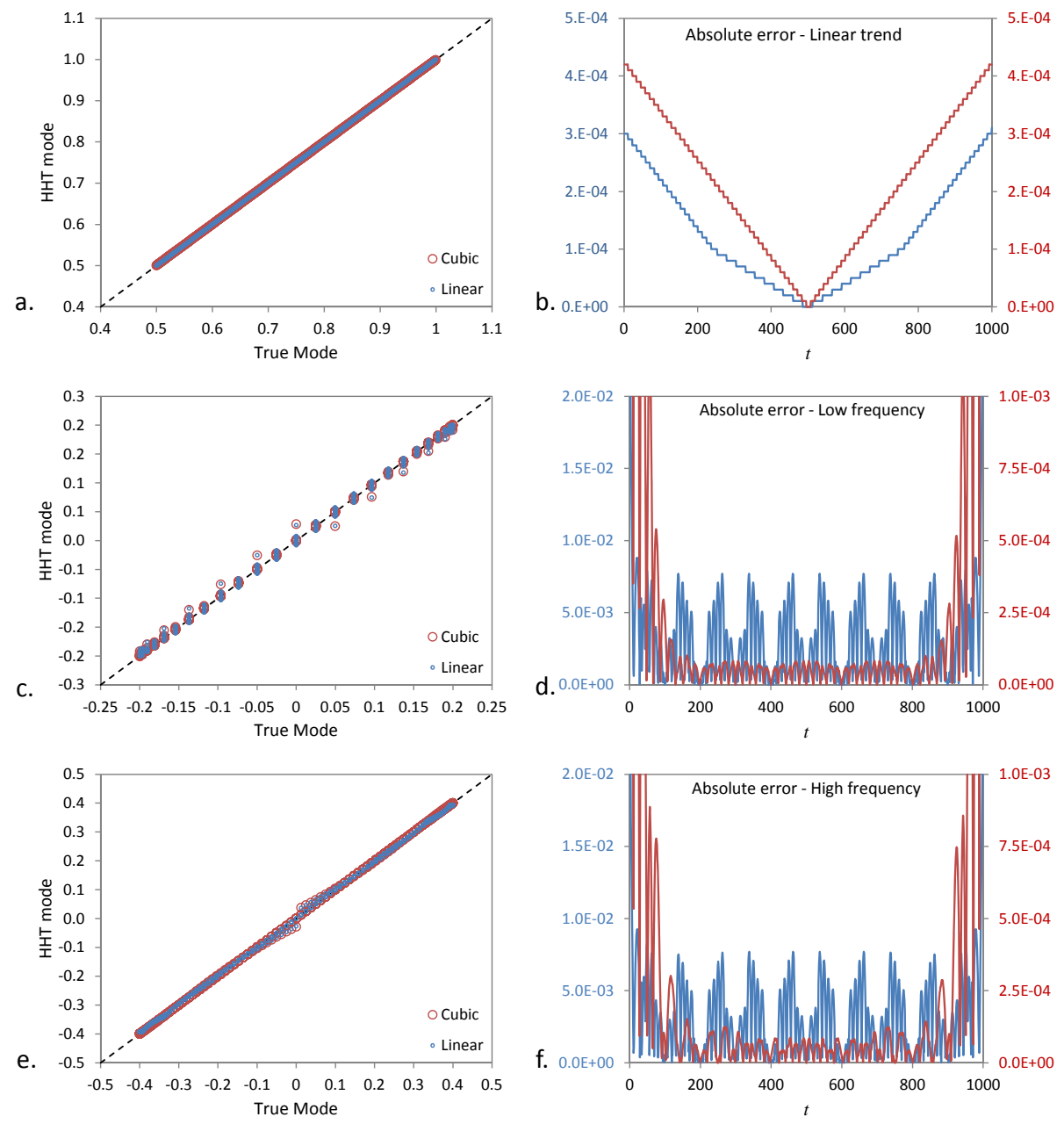

Figure 2. Results for the synthetic data (1). In all figure panels, results pertaining to the linear EMD method are in blue and those pertaining to the cubic EMD method are in red: (a) EMD/HHT trend plotted against the actual trend; (b) Absolute error between modelled and actual trend; (c) EMT/HHT low frequency signal plotted against the actual low frequency component; (d) Absolute errors for the low frequency component; (e) EMT/HHT high frequency signal plotted against the actual high frequency component; (d) Absolute errors for the high frequency component.

equal to $10 \%$ of the standard deviation of the input data and a 50 member ensemble. The linear and cubic EEMD methods both do a reasonable job of reproducing the linear trend in the signal (Figure 1a), though it appears that the linear EEMD method is marginally more accurate. This is confirmed by Figure 1b, which shows that the absolute errors for the linear EEMD method were generally lower than those for the cubic EEMD method. The mean absolute error for the linear EEMD method was $5.8 \times 10^{-3}$, compared to $1.3 \times 10^{-2}$ for the cubic EEMD method.

Figures $1 \mathrm{c}$ and $1 \mathrm{~d}$ display the corresponding results for the low frequency signal component. In this instance the linear EEMD and cubic EEMD methods perform more poorly, with the linear EEMD mode clearly effected by mode mixing issues. specifically, the oscillations in the linear EEMD trace in Figure 1c is due to mixing of the high frequency signal component in the EEMD mode. The cubic EEMD trace in Figure 1c is also corrupted by mode mixing though its effect is not as obvious. Figure 1d indicates that the linear EEMD method produced roughly twice the absolute error of the cubic EEMD method with a mean absolute error of $1.8 \times 10^{-1}$ compared to $7.0 \times 10^{-2}$ for the cubic EEMD method.

The results for the high frequency signal component, shown in Figure 1e and $1 \mathrm{f}$ indicate that both the linear and cubic EEMD methods did a reasonable job of reproducing the true high frequency component. The absolute errors in Figure 1f suggest that there is little difference in the two methods except at the ends of the domain where the cubic EEMD method tends to produce significantly larger absolute errors. The mean absolute error 
F.M. Ziaeyan Bahri and J.J. Sharples, Sensitivity of the Hilbert-Huang Transform to interpolation methodology...

for the high frequency component was $3.0 \times 10^{-2}$ using linear interpolation, and $3.1 \times 10^{-2}$ using cubic interpolation.

\subsection{Sea level data}

The linear and cubic EEMD methods were applied to the Brisbane sea level data to determine a long term trend. The method was again applied by adding a noise component with standard deviation equal to $10 \%$ of the standard deviation of the input data and a 50 member ensemble. The results, presented in Figure 3 , indicate significant differences in the long term trend behaviour estimated by the two methods. In particular the linear EEMD method exhibits a non-monotonic trend that possesses a discontinuous derivative. Differences in the two long term trend estimates are most significant at the ends of the domain, where they differ by approximately $5 \mathrm{~cm}$. While this may not seem much in absolute terms, it is important to note that when dealing with the ramifications of global and regional sea level change, trends of the order of millimetres per decade can be important, and so the differences encountered here could be quite significant in applications. Moreover, without knowing what the actual trend in the sea level data set is, it is difficult to assess which trend estimate is the more faithful. Indeed both the cubic and linear EEMD trends appear to reproduce the general behaviour of the time series reasonably well.

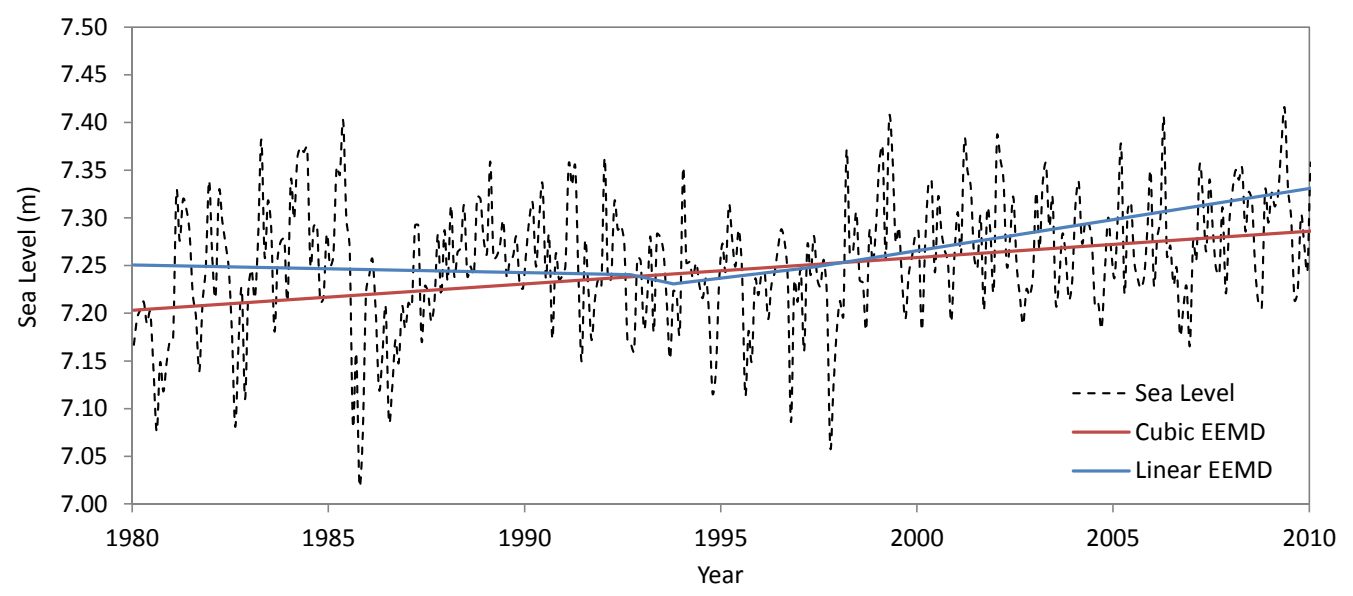

Figure 3. Brisbane sea level time series overlayed with estimates of the long term trend as determined by the cubic EEMD method (red) and the cubic EEMD method (blue).

\section{DISCUSSION AND CONCLUSIONS}

This paper has examined the effect of replacing the cubic interpolation methodology that is routinely employed in the EMD procedure with a linear interpolation methodology. When applied to noiseless synthetic data both the linear and cubic EMD procedures were found to reproduce the component signals quite well. On average the linear EMD method provided better estimates of the linear trend component of the input signal, while the cubic EMD method provided better estimates of both the low and high frequency oscillatory components of the signal. When applied to the noisy synthetic data, the linear EEMD method again provided the better estimate of the trend component, while the cubic EEMD method provided the better estimate of the low frequency component. For the high frequency component in the noisy synthetic data set, the linear and cubic EEMD methods produced almost the same average errors, with the linear EEMD method slightly more accurate. It should be noted that for the noisy synthetic data set both the linear and cubic EEMD methods suffered from mode mixing problems.

For the real geophysical data set of sea level at Brisbane, the linear and cubic EEMD methods gave different estimates of the long term trend. The higher frequency ensemble IMFs delivered by the linear and cubic EEMD procedures (not shown) were quite similar, but the separate ensemble IMFs became more disparate as the frequency decreased. In the absence of a thorough analysis of the significance of the estimated trends, the differences found in the trend estimates provided by the linear and cubic based EEMD methods raise some questions about the overall applicability of the HHT, in addition to those that have been raised by other authors (Dätig and Schlurmann, 2004; Deering and Kaiser, 2005). 
F.M. Ziaeyan Bahri and J.J. Sharples, Sensitivity of the Hilbert-Huang Transform to interpolation methodology...

There are of course a wide array of interpolation methods that could be used instead of linear or cubic interpolation methods; for example, smoothing splines. Examining the effects of further variation in interpolation methodology, as well as the significance of the component estimates, will form the basis for further work.

\section{ACKNOWLedgement}

The authors are grateful to Tal Ezer from the Old Dominion University for the provision of Matlab code used to implement the empirical mode decomposition. J.J. Sharples is supported by the Australian Research Council (IN130100038, IN140100011).

\section{REFERENCES}

Church, J. A., J. R. Hunter, K. L. McInnes, and N. J. White (2006). Sea-level rise around the australian coastline and the changing frequency of extreme sea-level events. Australian Meteorological Magazine 55(4), 253-260.

Dätig, M. and T. Schlurmann (2004). Performance and limitations of the hilbert-huang transformation (hht) with an application to irregular water waves. Ocean Engineering 31(14), 1783-1834.

Deering, R. and J. F. Kaiser (2005). The use of a masking signal to improve empirical mode decomposition. In Acoustics, Speech, and Signal Processing, 2005. Proceedings.(ICASSP'05). IEEE International Conference on, Volume 4, pp. iv-485. IEEE.

Ezer, T., L. P. Atkinson, W. B. Corlett, and J. L. Blanco (2013). Gulf stream's induced sea level rise and variability along the us mid-atlantic coast. Journal of Geophysical Research: Oceans 118(2), 685-697.

Hahn, S. L. (1996). Hilbert Transforms in Signal Processing. Artech House on Demand.

Huang, N. E., C. C. Chern, K. Huang, L. W. Salvino, S. R. Long, and K. L. Fan (2001). A new spectral representation of earthquake data: Hilbert spectral analysis of station tcu129, chi-chi, taiwan, 21 september 1999. Bulletin of the Seismological Society of America 91(5), 1310-1338.

Huang, N. E., Z. Shen, S. R. Long, M. C. Wu, H. H. Shih, Q. Zheng, N.-C. Yen, C. C. Tung, and H. H. Liu (1998). The empirical mode decomposition and the hilbert spectrum for nonlinear and non-stationary time series analysis. In Proceedings of the Royal Society of London A: Mathematical, Physical and Engineering Sciences, Volume 454, pp. 903-995. The Royal Society.

Huang, N. E. and Z. Wu (2008). A review on hilbert-huang transform: Method and its applications to geophysical studies. Reviews of Geophysics 46(2).

Peel, M., T. McMahon, and G. Pegram (2009). Assessing the performance of rational spline-based empirical mode decomposition using a global annual precipitation dataset. Proceedings of the Royal Society of London A 465, 1919-1937.

Peel, M., T. McMahon, R. Srikanthan, and K. Tan (2011). Ensemble empirical mode decomposition: testing and objective automation. In Proceedings of the 34th World Congress of the International Association for Hydro-Environment Research and Engineering: 33rd Hydrology and Water Resources Symposium and 10th Conference on Hydraulics in Water Engineering, pp. 702. Engineers Australia.

Pegram, G., M. Peel, and T. McMahon (2008). Empirical mode decomposition using rational splines: an application to rainfall time series. Proceedings of the Royal Society of London A 464, 1483-1501.

Rahmstorf, S. (2012). Sea-level rise: towards understanding local vulnerability. Environmental Research Letters 7(2), 021001.

Rilling, G., P. Flandrin, P. Goncalves, et al. (2003). On empirical mode decomposition and its algorithms. In IEEE-EURASIP Workshop on Nonlinear Signal and Image processing. NSIP-03, Grado.

Salisbury, J. and M. Wimbush (2002). Using modern time series analysis techniques to predict enso events from the soi time series. Nonlinear Processes in Geophysics 9(3/4), 341-345.

Srikanthan, R., M. Peel, T. McMahon, and D. Karoly (2011). Ensemble empirical mode decomposition of australian monthly rainfall and temperature data. In Proceedings of the 19th International Congress on Modelling and Simulation, Perth, WA, Australia, Volume 1216, pp. 36433649. 